\title{
Color-tunablility in GaN LEDs Based on Atomic Emission Manipulation Under Current Injection
}

Brandon Mitchell ${ }^{1,2}$, Ruoqiao Wei ${ }^{3}$, Junichi Takatsu ${ }^{2}$, Dolf Timmerman ${ }^{2}$, Tom Gregorkiewicz ${ }^{2,4}$, Wanxin $\mathrm{Zhu}^{2}$, Shuhei Ichikawa, ${ }^{2}$ Jun Tatebayashi ${ }^{2}$, Yasufumi Fujiwara ${ }^{2}$, and Volkmar Dierolf ${ }^{3}$

${ }^{1}$ Department of Physics, West Chester University, West Chester, PA, 19383, USA

${ }^{2}$ Division of Materials and Manufacturing Science, Graduate School of Engineering, Osaka University, 21 Yamadaoka, Suita, Osaka 565-0871, Japan

${ }^{3}$ Department of Physics, Lehigh University, Bethlehem, Pennsylvania 18015, USA

${ }^{4}$ Van der Waals-Zeeman Institute, University of Amsterdam, Science Park 904, 1098 XH Amsterdam, The Netherlands

One page and one figure. 


\section{SUPPORTING INFORMATION}

\section{RGB properties of the RB-LED.}

The RB-LED was able to produce blue emission from defect levels associated with the Si-Mg. In addition, some Eu centers were enhanced by the presence of local $\mathrm{Si}$ and $\mathrm{Mg}$ atoms,[33] but were few in number and saturated quickly, leaving the rest of the Eu centers able to be excited for higher injection currents. Because the other Eu centers are simply the typical centers found in $\mathrm{GaN}: \mathrm{Eu}$, these centers can be re-excited and produce yellow luminescence, but not blue luminescence. Note: the Mg-Si centers can also likely produce yellow luminescence, but is difficult to separate it from the other Eu centers. At high injection currents ( $\sim 90 \mathrm{~mA})$, the RB-LED emits a white-pink, while at low injection currents $(\sim 40 \mathrm{~mA})$ it emits a bluer magenta. In Fig. S1, images of the LED under these conditions are shown along with filtered images to show the underlying colors. By using a $600 \mathrm{~nm}$ short-pass and $600 \mathrm{~nm}$ long-pass filter for the lower current case, yields the blue and red emission, respectively. At a higher current, using the 600nm short pass filter alone does not show blue emission, but rather teal emission. By using a $470 \mathrm{~nm}$ long-pass filter in addition to the $600 \mathrm{~nm}$ short-pass filter (blocking both the ${ }^{5} \mathrm{D}_{0}$ and NBE emission) yields a green-yellow emission from the ${ }^{5} \mathrm{D}_{1}$ state. Thus, the white-pink is a result of mixing red, green and blue, but where the red emission contribution is too high to produce white light resulting in a very unsaturated red.
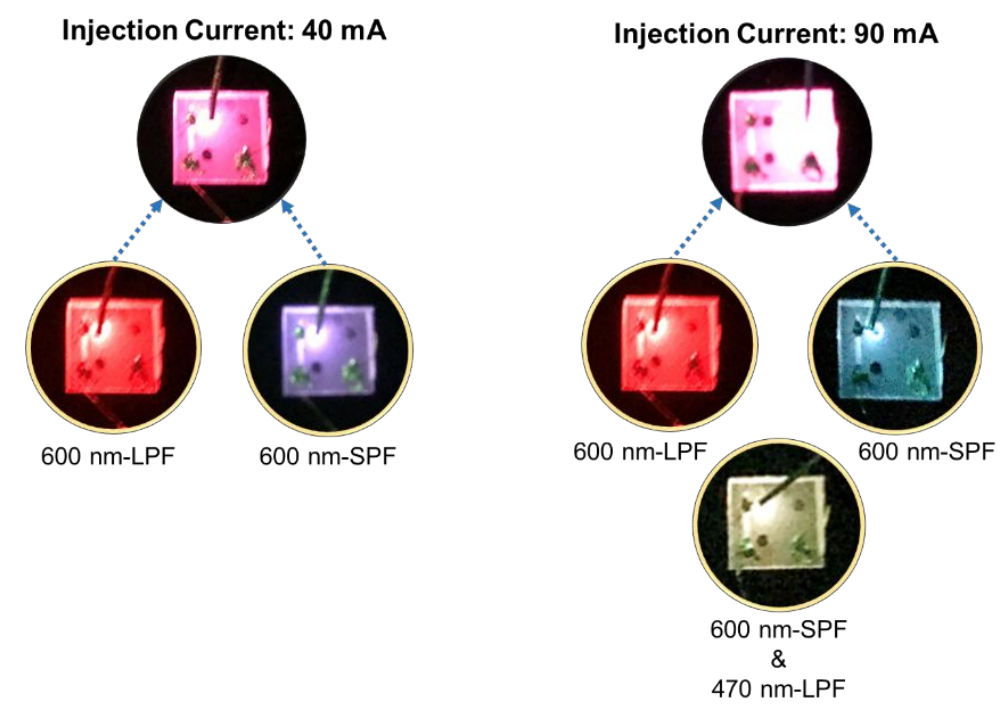

Figure S1 | Images of the RB-LED under different current injection and filtering conditions.

Images of the RB-LED under constant injection currents of $40 \mathrm{~mA}$ and $90 \mathrm{~mA}$. The unfiltered images of the LEDs are shown at the top for both conditions. Images of these LEDs with various filters are shown below the images. 